\title{
Symptomatic Parkinsonism*
}

\author{
JoHn Pearce \\ M.D., F.R.C.P. \\ Department of Neurology, Hull Royal Infirmary
}

\begin{abstract}
Summary
Parkinson's disease, 'paralysis agitans', is characterized by a number of abnormalities.

A similar clinical picture may be symptomatic of other disturbances: these are briefly described and the differential diagnosis is discussed.
\end{abstract}

\section{Introduction}

The characteristic features of paralysis agitans have long been familiar to doctors in many disciplines. For the most part the diagnosis poses no serious strain on the doctor's intellect or powers of observation. The typical resting tremor, cog-wheel and plastic rigidity in the neck, trunk and limbs accompanied by the important disorder of posture and the poverty and slowness of volitional movement immediately attract attention. Diagnosis is almost entirely based on clinical observation. It is frequently made within a few seconds of the patient's entry to the consulting room, and if the diagnostic impression is not formed at this stage it may escape recognition for some considerable time.

Present day practice yields a population of the idiopathic type of disease as originally described by James Parkinson in 1817 , in over $90 \%$ of patients. Practice in a mental hospital of course discloses a much higher proportion of patients afflicted by druginduced disease, which will be discussed later. By idiopathic, we imply that the primary aetiological agent is unknown. A depletion of dopaminergic central neuro-transmission (Bernheimer et al., 1973) operating mainly at the level of the substantia nigra and its efferent pathway the corpus striatum is the fundamental biochemical basis which is probably common to all patterns of Parkinsonism. It is, however, clear that dopamine deficiency is not the sole basis of the disease, for were this so, it would be arrested or cured in every case by replacement of dopamine by various pharmacological manoeuvres. The failures and relatively short-term success of dopamine replacement are sufficiently obvious to indicate that there is much more involved than a simple deficiency of this transmitter substance.

\footnotetext{
* Paper presented at a Trent Regional Symposium on Parkinson's disease held at Doncaster Royal Infirmary on April 24 1976. Edited by Dr A. A. G. Lewis, and received for publication June 131977.
}

Nevertheless, depletion of the pigmented nerve cells $\overrightarrow{-}$ in the substantia nigra is an essential ingredient of ${ }_{-}^{\circ}$ both the idiopathic and the much less common $\vec{\omega}$ symptomatic varieties of Parkinsonism. In addition? to the nerve cell loss and formation of Lewy bodieso in Parkinson's disease, there is also frequently found $\overline{\text {. }}$. a degree of reduction of brain weight, dilatation of $\mathrm{c}$ the ventricular system and widening of the corticalio sulci. Similar changes have been found in post- encephalitic varieties of disease, and are also N constantly observed in the parkinsonian syndrome associated with Alzheimer's pre-senile dementia to which reference will be made later.

\section{Physical agents}

Before coming to the more commonly encountered 3 syndromes, some rare causes of the syndrome must be mentioned. Firstly, instances of mangane $e_{0}$ poisoning and carbon monoxide poisoning figure $\mathrm{n}$ all the major neurological treatises. They afe extremely rare in clinical practice, but both agentso are known to damage nigrostriatal neurones by cytotoxic mechanisms.

\section{Trauma}

Trauma is frequently invoked as a possible cause $\overrightarrow{\vec{O}}$ or aggravating agent in Parkinsonism. A history of 3 trauma is given by about $10 \%$ of patients with Parkinson's disease, but from this we should not? assume that it is a causative factor. Examples of aggravation of previously mild Parkinsonism by a. head injury are occasionally encountered in practice, $\frac{3}{3}$ and more rarely one sees a patient with no previous history or evidence of symptoms of the disease who윽 develops them in a dramatic fashion shortly after a head injury. The contentious argument rests on옹 whether the patient had the disease in a latent form? before the injury which then acted as an aggravating? factor, or whether the brain damage initiated the N disease de novo. There is virtually no evidence to confirm that trauma can cause the disease. But, in 0 medico-legal practice one sees a variety of un- $\omega$ doubtedly organic sequelae of mild to moderate? head injuries in elderly subjects which one would seldom encounter in the younger brain. Amongst $\Phi$ these, Parkinsonism features rarely, but when it does ${ }^{-}$ it is reasonable to assume that trauma played a minor ${ }^{\circ}$ 
role as a precipitating or aggravating factor. It is striking that in the numerous head injuries encountered in war-time, Parkinsonism was virtually never encountered. Finally, to prove that trauma was a causal factor, it would be necessary to show that the chances of acquiring the disease are greater among the injured portion of the population than among the uninjured: no supporting figures have ever been adduced to support this concept.

\section{Cerebral tumour}

Brain tumours are amongst the rarest causes of symptomatic Parkinsonism. They almost invariably produce their own characteristic combination of focal signs, raised intracranial pressure, epilepsy or mental disturbance. One has occasionally met patients with long-standing Parkinson's disease of ten or more years' duration who have then developed a brain tumour, usually a glioma and one has regarded this as coincidental. Occasionally, however, a parasagittal meningioma or glioma of the corpus callosum or thalamus can present with Parkinsonism (Marguilies, 1953). The course of the illness is relentlessly downhill and the speed of progression far greater than in idiopathic paralysis agitans. These anomalous clinical features will usually direct attention to the need for further investigation.

\section{Commoner varieties of symptomatic Parkinsonism \\ Juvenile paralysis agitans}

Hunt in 1917 reported a case of Parkinsonism beginning at the age of 15 and going on for 25 years and found lesions of the basal ganglia at postmortem. Many similar cases have subsequently been described and are referred to as juvenile paralysis agitans. Lesions in the substantia nigra and pallidum have been observed. The author has personally encountered eight examples of the syndrome which started before the age of 40 including one case starting at 16 and three before the age of 30 . Perhaps the most striking feature of this syndrome is that although the disease appears fully developed with tremor, rigidity and bradykinesia, it seems to advance for a number of years and then remains relatively stationary. The author's impression is that the natural history is considerably longer than in the later onset idiopathic disease. Wilson (1940) thought it doubtful that these cases really belonged to the group of classical Parkinson's disease.

\section{Post-encephalitic disease}

The well known epidemic of encephalitis which started in Austria and France in 1916 and travelled round the world left in its wake the complication of Parkinsonism in a substantial number of patients. Among 925 encephalitics, $334(36 \%)$ manifested post- encephalitic Parkinsonism in greater or lesser degree (Parsons, 1928). The syndrome would develop during the convalescent stage of the illness, or more frequently in the next 12 to 18 months. In rare instances, a delay of up to 10 years was recorded. To the classic features of rigidity, tremor, dysarthria and flexion dystonia, were added tics and twitches of the face, throat and jaw, attacks of myoclonus and, most characteristic of all, oculogyric crises. Other than the oculogyric crises, an early age of onset and a definite history of encephalitis, there were no diagnostic features. Tremor tended to be less frequent, stiffness more marked. Excessive salivation was commoner and muscular weakness rarer. Loss of ocular convergence, pupillary abnormalities and marked asymmetry were typical but not diagnostic features. In the more severe cases the prognosis was worse than in paralysis agitans, but the fact that there is still a large number of survivors in whom the disease has apparently become stationary after the passage of many years, indicates that at least one variant of this extraordinary condition is comparatively benign. In passing, it is of interest that these patients have been unusually sensitive to dopamine replacement, and that oculogyric crises are usually unaffected by this treatment.

\section{'Arteriosclerotic Parkinsonism'}

We are all familiar with the cases of bilateral hemiplegia or patients who have sustained multiple minor strokes. The latter group are often hypertensive without major extracranial vascular disease but with a multitude of tiny lacunae which represent microinfarcts in the basal ganglia internal capsule and pons. The sliced brain in such a case in the affected region resembles Gruyère cheese. The clinical features of these patients are characteristic, and include bilateral spastic weakness of face, arms and legs, often asymmetrical with enhanced reflexes and extensor plantar responses. Explosive laughter and crying, dysphagia and a highly characteristic nasal dysarthria, and a brisk jaw-jerk constituting 'pseudobulbar palsy' complete the picture. The bilateral spastic weakness of the facial muscles produces an immobile expressionless appearance, and the involvement of pyramidal tracts in both legs produces a slow shuffling, short-stepped gait. The superficial resemblance to Parkinson's disease is thus evident. But the resemblance is only superficial and the true extra-pyramidal signs of basal ganglia disease are seldom seen (Pearce and Pearce, 1971). It is of course true that the patients with Parkinsonism in their 70's and 80 's will frequently have co-existing cerebrovascular disease, but all the current evidence suggests that they are purely coincidental. Eadie and Sutherland (1964) suggested that the incidence of arterial disease in parkinsonian patients did not differ from control 
subjects of similar age and sex distribution. Finally, direct pathological correlation between Parkinsonism and discrete cerebrovascular lesions is rare.

\section{Drug-induced Parkinsonism}

Parkinsonism induced by drug therapy is now the commonest form of symptomatic disease. In 1961, Ayd found that of 3775 patients treated with drugs of the phenothiazine group, 1472 had extra-pyramidal syndromes. Of these, $21.2 \%$ showed akathisia, $15.4 \%$ Parkinsonism, and $2.3 \%$ dyskinesia including oculogyric crises. One can therefore expect to find parkinsonian signs in about $33 \%$ of all patients with long-term phenothiazine treatment. Whether the disorder can be wholly prevented by concomitant medication with anticholinergic drugs remains doubtful, but it is certain that symptoms and disability are considerably lessened by such therapy.

How long it takes to induce Parkinsonism with psychotropic drugs is clinically relevant. It has been observed between 3 and 6 weeks after treatment of tetanus with chlorpromazine. Under these circumstances of relatively brief courses of therapy the syndrome is entirely reversible when treatment is stopped. There is, however, evidence that after continued treatment for many months or years, withdrawal of the drug may, on occasion, not be followed by the remission which usually occurs within 2 or 3 weeks of its discontinuation.

Reserpine exerts its effect by displacing dopamine from nerve endings, and in this instance levodopa will reverse the iatrogenic syndrome. Phenothiazines and butyrophenones including haloperidol act by causing blockade of the dopamine receptors, and in these patients the beneficial effect of levodopa is less striking, and a better response may be obtained by anticholinergic drugs.

In all instances of apparent drug-induced Parkinsonism, the clinical problem is whether the syndrome is entirely iatrogenic or whether the patient harboured latent Parkinson's disease which has become manifest only by further dopamine antagonism caused by the drug therapy. It may be argued that drug-induced cases which do not revert to normality on withdrawal of the drug are in fact examples of latent Parkinson's disease which has become unmasked by the psychotropic agent.

\section{Alzheimer's disease}

Although dementia is the most striking ingredient of Alzheimer's disease, a variety of subtle neurological signs are almost invariably found in this syndrome. A tetrad of clinical features has been described which becomes relevant in this discussion because of the high incidence $(60 \%)$ of a Parkinsonian syndrome (Pearce, 1974). The tetrad consists of:
1. Dementia. 2. Parkinsonian signs. 3. Primitive reflexes, (a) glabellar tap, (b) snout and sucking $\frac{\mathbb{Q}}{\square}$ (c) grasp, (d) palmo-mental. 4. Cerebral cortical and-. central atrophy (Selby, 1967).

The incidence of Parkinsonism in the context op dementia is of some interest, and is accompanied by histological changes in the basal ganglia in primary Alzheimer's disease (Pearce, 1974). The interactions of motor cortex and basal ganglia are complex. Then cortical lesion may cause a supranuclear denervation of basal nuclei. Conversely, basal ganglia disease can cause dysfunction of the cortex by interruption of $\overrightarrow{\tilde{w}}$ proprioceptive pathways. In this context it is of interest that depletion of monoamines in the cortex has recently been shown in senile dementia.

\section{Conclusions}

With the exception of drug-induced disease symptomatic Parkinsonism is relatively uncommon in clinical practice. Its theoretical implications rangeo widely as has been suggested in this somewhat sketchy survey of the field. When confronted with (i) atypical clinical signs; (ii) a syndrome whose pro- $-\frac{\mathbb{R}}{8}$ gression is unusual; (iii) a response to treatment ${ }^{\mathbb{D}}$ which does not follow the expected course, clinician would be wise to reassess the patient apd investigate further to clarify the possibility $\Phi f \overrightarrow{0}$ Parkinsonism secondary to some other primây. disease process.

\section{References}

AYD, J. (1961) A survey of drug induced extrapyramidalo reactions. Journal of the American Medical Association, $175,1054$.

BERNHEIMER, H., BIRKMAYER, W., HoRNYKIEWICZ, O., $\overrightarrow{\overrightarrow{\overline{0}}}$ Jellinger, K. \& Seitelberger, F. (1973) Brain dopamine and the syndromes of Parkinson and Huntington. Clinical, morphological and neurochemical correlations. Journal of Neurological Sciences, 20, 415.

Eadie, M.J. \& Sutherland, J.M. (1964) Arteriosclerosis in Parkinsonism. Journal of Neurology, Neurosurgery and. Psychiatry, 27, 237.

HuNT, J.R. (1917) Progressive atrophy of the globus pallidus Brain, 40, 59. MARGUILIES, M.E. (1953) Parkinsonism and brain tumour.
Archives of Neurology and Psychiatry, 70, 112 .

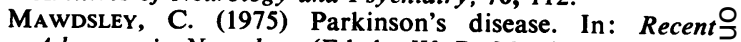
Advances in Neurology (Ed. by W. B. Matthews), p. 118. Churchill Livingstone, London, Edinburgh.

Parkinson, J. (1817) An Essay on the Shaking Palsy. Whittingham and Rolland, London.

PARSONS, A.C. (1928) Ministry of Health Reports 1949.

PeARCE, J. (1974) The extrapyramidal disorder of Alzheimer's $N$ disease. European Neurology, 12, 94. PeARCE, J. \& PeArCe, I. (1971) Current concepts of Parkin- $-\frac{\omega}{\sigma}$
sonism. Postgraduate Medical Journal, 47, 794 .

SELBY, G. (1967) Stereotactic surgery for relief of Parkinson's disease. 1. Critical review. Journal of the Neurological $\bar{\varnothing}$ Sciences, 5, 315.

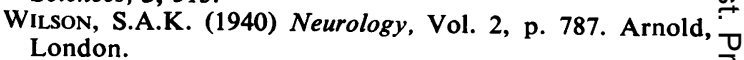

